\title{
Risk factors for low vitamin D status in Korean adolescents: the Korea National Health and Nutrition Examination Survey (KNHANES) 2008-2009
}

\author{
Young Ah Lee ${ }^{1}$, Hwa Young Kim ${ }^{1}$, Hyunsook Hong ${ }^{2}$, Ji Young Kim ${ }^{1}$, Hyun Jin Kwon ${ }^{3}$, \\ Choong Ho Shin ${ }^{1, *}$ and Sei Won Yang ${ }^{1}$ \\ 'Division of Endocrinology and Metabolism, Department of Pediatrics, Seoul National University Children's \\ Hospital, Seoul National University College of Medicine, 28 Yeongeon-dong, Jongno-gu, Seoul 11 10-769, \\ Republic of Korea: ${ }^{2}$ Medical Research Collaborating Center, Seoul National University Hospital, Seoul, \\ Republic of Korea: ${ }^{3}$ Health and Exercise Science, Institute of Sports Science, Seoul National University, \\ Seoul, Republic of Korea
}

Submitted 31 May 2012: Final revision received 17 January 2013: Accepted 22 January 2013: First published online 6 March 2013

\begin{abstract}
Objective: To evaluate the prevalence of vitamin D deficiency and predictors for low vitamin D status in Korean adolescents living between latitudes $33^{\circ}$ and $39^{\circ} \mathrm{N}$. Design: A descriptive cross-sectional study.

Setting: Korea National Health and Nutrition Examination Survey (KNHANES) 2008-2009.

Subjects: A total of 1510 healthy adolescents aged 12-18 years (806 male, mean age 14.7 years) participated. Possible predictors for low vitamin D status (logtransformed 25-hydroxyvitamin D (25(OH)D) concentrations) were evaluated. Results: The prevalence of vitamin D deficiency $(25(\mathrm{OH}) \mathrm{D}<20 \mathrm{ng} / \mathrm{ml})$ was $89.1 \%$ in spring, $53.7 \%$ in summer, $63.9 \%$ in autumn and $90.5 \%$ in winter. Winter season, older age, higher education level reached, being female, being obese, a lack of vitamin D supplementation, lower milk consumption $(0-<200 \mathrm{ml} / \mathrm{d})$ and a lack of physical activity were unadjusted predictors (all $P<0.05)$. Multiple linear regression analysis showed that winter season $(P<0 \cdot 001)$, higher education level $(P<0 \cdot 001)$ and a lack of vitamin D supplementation $(P=0 \cdot 012)$ were independent predictors for low vitamin D status. The modifying effect of season on the association between vitamin D supplement use and vitamin D status was significant $(P<0 \cdot 001)$.

Conclusions: Vitamin D deficiency was highly prevalent in Korean adolescents, especially those in higher school grades. Vitamin D supplementation may contribute to maintain a better vitamin D status with lower seasonal variation. Further studies are required to determine optimal vitamin D intakes to maintain sufficient vitamin D status for Korean adolescents.
\end{abstract}

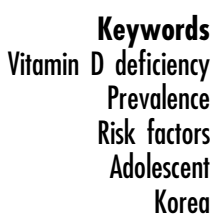

There is great interest in the role of vitamin D in multiple health outcomes. It is essential for skeletal health, and there has been increasing evidence linking vitamin D with benefits for non-skeletal outcomes such as type 2 diabetes mellitus, CVD, infectious/inflammatory disease, autoimmune disease and cancer ${ }^{(1)}$.

Although there has been much debate over the definition of vitamin D deficiency, most agree that a 25-hydroxyvitamin $\mathrm{D}(25(\mathrm{OH}) \mathrm{D})$ concentration of $<20 \mathrm{ng} / \mathrm{ml}$ is an indication of deficiency ${ }^{(2-5)}$. Using this definition, vitamin $\mathrm{D}$ deficiency is common in the USA ${ }^{(6-9)}$, New Zealand ${ }^{(10)}$, the $\mathrm{UK}^{(11)}$ and Beijing in China ${ }^{(12)}$. Recent data for the Korean population based on the Korea National Health and Nutrition Examination Survey (KNHANES) 2008 ${ }^{(13)}$ showed that vitamin D insufficiency was most prevalent in those aged 20-29 years, followed by those aged 10-19 years. In the adult population, predictors for vitamin $\mathrm{D}$ insufficiency included being in younger age groups, having $25(\mathrm{OH}) \mathrm{D}$ concentrations checked in spring and winter, living in an urban area and undertaking indoor occupations ${ }^{(13)}$. However, the predictors for vitamin D insufficiency in the paediatric population were not included.

There has been a paucity of data on the importance of vitamin D status for health outcomes as well as on the important predictors for deficiency in Korean adolescents. The Dietary Reference Intake (DRI) of vitamin D for Korean children and adolescents was lowered from $10 \mu \mathrm{g} / \mathrm{d}$ in 2005 to $5 \mu \mathrm{g} / \mathrm{d}$ in $2010^{(14)}$, under the assumption that sunlight exposure could satisfy their vitamin D needs. The purposes of the present study were to: (i) evaluate 
the prevalence of vitamin D deficiency in Korean adolescents aged 12-18 years based on the Fourth Korea National Health and Nutrition Examination Survey (KNHANES IV) in 2008-2009; and (ii) investigate the risk factors leading to low vitamin D status in Korean adolescents living between latitudes $33^{\circ}$ and $39^{\circ} \mathrm{N}$.

\section{Materials and methods}

\section{Study participants}

The present study is based on the data acquired during the second and third years (2008 and 2009) of KNHANES IV under permission of the Korea Centers for Disease Control and Prevention. KNHANES IV was a cross-sectional representative survey of the non-institutionalized population of Korea from 2007 to 2009. It consisted of a health interview survey, a nutrition survey and a health examination survey. KNHANES IV used a rolling sampling design with a complex, stratified and multistage probability sampling. Based on the 2005 National Census Registry for Korea, from among 264186 primary sampling units, 200 were selected randomly. Then, twenty-three households were sampled from each unit to yield 4600 households. In 2008 and 2009, 25250 individuals (12528 and 12722, respectively) were sampled; 19386 (9308 and 10078, respectively) of these individuals participated in health interviews and health examination surveys and 18038 (8641 and 9397, respectively) participated in nutrition surveys. Among 1933 participants aged 12-18 years, 423 were excluded; 354 had missing $25(\mathrm{OH}) \mathrm{D}$ values and sixty-nine were on medications and/or had a chronic disease possibly affecting vitamin D absorption and metabolism (e.g. congenital heart disease, epilepsy, jaundice and/or liver disease, renal disease, thyroid disorder, malignancy, or a psychiatric or bone disease related to immobilization). Finally, 1510 participants (806 boys and 704 girls) aged 12-18 years were included in the present study. KNHANES IV was conducted according to the guidelines laid down in the Declaration of Helsinki. All participants in the survey signed an informed consent form. The institutional review board of the Korea Centers for Disease Control and Prevention approved the protocol.

\section{Study variables}

Factors were categorized to analyse the risk factors for vitamin D deficiency. Korea is located in a temperate region with four distinct seasons: spring (March to May), summer (June to August), autumn (September to November) and winter (December to February). The education levels of the study participants aged $12-18$ years were categorized into 5th and 6th grades (elementary school, $n$ 123), 7 th to 9th grades (middle school, $n 733$ ), 10th to 12th grades (high school, $n 546$ ) and first collegiate year ( $n 57)$. This was based on the assumption that each group would have a different level of outdoor activity and sunlight exposure. BMI percentiles and BMI Z-scores were assigned on the basis of the 2007 Korean National Growth Charts $^{(15)}$ and the participants were classified into three groups according to BMI percentile: normal weight $(<85$ th percentile), overweight (85th- $<95$ th percentile) and obese ( $\geq 95$ th percentile).

Any dietary vitamin D supplementation was indicated as 'yes' when the participants consumed any supplementation. This 'yes' group was subcategorized into two groups according to the amount of supplementation: $\geq 5 \mu \mathrm{g} / \mathrm{d}$ and $0-<5 \mu \mathrm{g} / \mathrm{d}$. Milk consumption data were obtained from the diet, behaviour and nutrition section of the NHANES Sample Person Questionnaire and were categorized as $0-<200 \mathrm{ml} / \mathrm{d}, 200-<400 \mathrm{ml} / \mathrm{d}$ and $\geq 400 \mathrm{ml} / \mathrm{d}$. Dietary and supplemental $\mathrm{Ca}$ intakes were compared with the DRI for Ca of Korean children and adolescents: $1000 \mathrm{mg} / \mathrm{d}$ for boys aged 12-14 years, $900 \mathrm{mg} / \mathrm{d}$ for girls aged 12-14 years, $900 \mathrm{mg} / \mathrm{d}$ for boys aged $15-18$ years and $800 \mathrm{mg} / \mathrm{d}$ for girls aged $15-18$ years ${ }^{(14)}$. Ca intake levels were categorized into two groups: $\geq$ DRI and $<$ DRI.

Participants were asked about their level of physical activity during a normal week. The definition of regular physical activity was based on the physical activity guideline provided by the US Department of Health and Human Services ${ }^{(16)}$ and output indicators of the Korea Youth Risk Behavior Web-based Survey ${ }^{(17)}$. Participants who performed moderate or vigorous physical activity for at least $60 \mathrm{~min} / \mathrm{d}$ on $3 \mathrm{~d} /$ week, and/or walking activity for at least $60 \mathrm{~min} / \mathrm{d}$ on $5 \mathrm{~d} /$ week, and/or muscular strength activity on more than $3 \mathrm{~d} /$ week were assigned to the 'regular physical activity' group. Those participants who performed moderate or vigorous physical activity for at least $180 \mathrm{~min} / \mathrm{d}$ on $3 \mathrm{~d} /$ week were also assigned to the 'regular physical activity' group. Regular physical activity was recorded as 'yes', regardless of whether it took place indoors or outdoors.

The style of dress in Korea is westernized and generally conservative. When the weather gets warmer in May with temperatures greater than $17-18^{\circ} \mathrm{C}$, the arms and legs are usually exposed in short-sleeved shirts and shorts or skirts. At the end of September when temperatures fall below $15-16^{\circ} \mathrm{C}$, long-sleeved shirts and trousers are worn. The face and neck are usually exposed except during cold winter days when temperatures approach $0^{\circ} \mathrm{C}$ and scarves are used to cover up. In middle school and high school, uniforms are the norm, whereas the attire of elementary and college students is not regulated. However, there is little difference in the degree of skin exposure according to type of dress between the groups. Thus, the type of dress was not included as a possible predictor.

\section{Measurement of serum 25-bydroxyvitamin $D$ concentration}

Blood samples were obtained by venepuncture, refrigerated immediately and transported to the central testing institute. 
Blood samples were analysed within $24 \mathrm{~h}$ after transportation. Serum 25(OH)D concentrations were measured using a gamma-counter (1470 Wizard; Perkin-Elmer, Turku, Finland) with a 25-hydroxyvitamin D ${ }^{125}$ I RIA kit (DiaSorin Inc., Stillwater, MN, USA). At serum 25(OH)D concentrations of $8 \cdot 6,22 \cdot 7,33 \cdot 0$ and $49 \cdot 0 \mathrm{ng} / \mathrm{ml}(21.5$, $56 \cdot 8,82.5$ and $122.5 \mathrm{nmol} / \mathrm{l})$, the inter-assay $\mathrm{CV}$ were $11.7 \%, 10 \cdot 5 \%, 8 \cdot 6 \%$ and $12.5 \%$, respectively, and the intra-assay $\mathrm{CV}$ were $9 \cdot 4 \%, 8 \cdot 2 \%, 9 \cdot 1 \%$ and $11 \cdot 0 \%$, respectively. KNHANES participates in the Vitamin D Standardization Program, so the measurement of $25(\mathrm{OH}) \mathrm{D}$ was standardized with the recently developed National Institute of Standards and Technology-Ghent University reference procedure ${ }^{(18)}$

\section{Statistical analysis}

Statistical analyses were conducted using the SAS statistical software package version 9·2. Data were analysed using several SAS survey procedures employing appropriate KNHANES sample weights to account for the complex KNHANES sampling design. The procedures included unequal probabilities of selection, oversampling and non-response so that inferences could be made about the Korean adolescent participants. The log-transformed $25(\mathrm{OH}) \mathrm{D}$ concentrations were found to be distributed normally in a Q-Q plot. Participants' characteristics were described using means and standard errors for continuous variables and numbers and percentages for categorical variables.

Participants' characteristics were calculated by quartile categories of serum 25(OH)D concentration $(<12$, $12-<16,16-<20$ and $\geq 20 \mathrm{ng} / \mathrm{ml}$ ). The Rao-Scott $\chi^{2}$ test was used to analyse associations between categorical variables and quartiles of serum $25(\mathrm{OH}) \mathrm{D}$ concentration. We estimated means with their standard errors for age, height, BMI $Z$-score and serum alkaline phosphatase levels according to quartiles of serum $25(\mathrm{OH}) \mathrm{D}$ concentration, and examined these associations with a test for linear trend.

We performed unadjusted linear regression analyses to investigate the relationship between possible predictors and vitamin D status (log-transformed 25(OH)D concentrations). The assumptions of our linear regression models were evaluated using normal probability plots of the standardized residuals and scatter plots with standardized residuals $v$. standardized predicted values. The assumptions were satisfied. Because the effect of predictors on vitamin D status might vary depending on the season, the modifying effect of season on the association between vitamin D status and each predictor was evaluated using a test for interaction. Predictors with $P<0 \cdot 2$ in the univariate analyses and interaction effects with $P<0.05$ were considered in the multiple linear regression analyses. Finally, multiple linear regression models were constructed for each season. Bonferroni-adjusted $P$ values were reported for multiple linear regression models.
For all analyses, $P<0.05$ (two-sided) was considered statistically significant.

\section{Results}

The study participants' (53.4\% male) mean age was $14 \cdot 7$ (sD 1.9) years and their mean BMI $Z$-score was 0.025 (SD $1 \cdot 05$ ), with $12 \cdot 2 \%$ of them being overweight and $5 \cdot 8 \%$ obese. Figure 1 demonstrates the proportion of participants with serum $25(\mathrm{OH}) \mathrm{D}$ concentrations of $<10$, $10-<15,15-<20,20-<30$ and $\geq 30 \mathrm{ng} / \mathrm{ml}(25,25-<37 \cdot 5$, $37 \cdot 5-<50,50-<75$ and $\geq 75 \mathrm{nmol} / 1)$ according to season. The prevalence of vitamin $\mathrm{D}$ deficiency, defined as a $25(\mathrm{OH})$ D level of $<20 \mathrm{ng} / \mathrm{ml}$, was $89 \cdot 1 \%$ in spring, $53 \cdot 7 \%$ in summer, $63.9 \%$ in autumn and $90.5 \%$ in winter.

Table 1 shows the associations between the quartile categories stratified according to serum 25(OH)D level and the participants' characteristics. Across the $25(\mathrm{OH}) \mathrm{D}$ quartile categories, the participants in the highest $25(\mathrm{OH}) \mathrm{D}$ group were significantly younger $(P<0 \cdot 001)$ and more physically active $(P=0.031)$ than those in the lowest $25(\mathrm{OH}) \mathrm{D}$ group. With increasing school grade, the serum $25(\mathrm{OH}) \mathrm{D}$ quartiles decreased significantly $(P<0 \cdot 001)$. Significantly increasing trends for serum 25(OH)D were observed with increasing vitamin $\mathrm{D}$ supplement use $(P=0 \cdot 028)$ and increasing milk consumption $(P=0 \cdot 002)$. As the $25(\mathrm{OH}) \mathrm{D}$ quartiles decreased, the level of serum alkaline phosphatase increased $(P<0 \cdot 001$; Table 1$)$.

Table 2 shows the unadjusted predictors for low vitamin D status, with log-transformed $25(\mathrm{OH}) \mathrm{D}$ concentration as

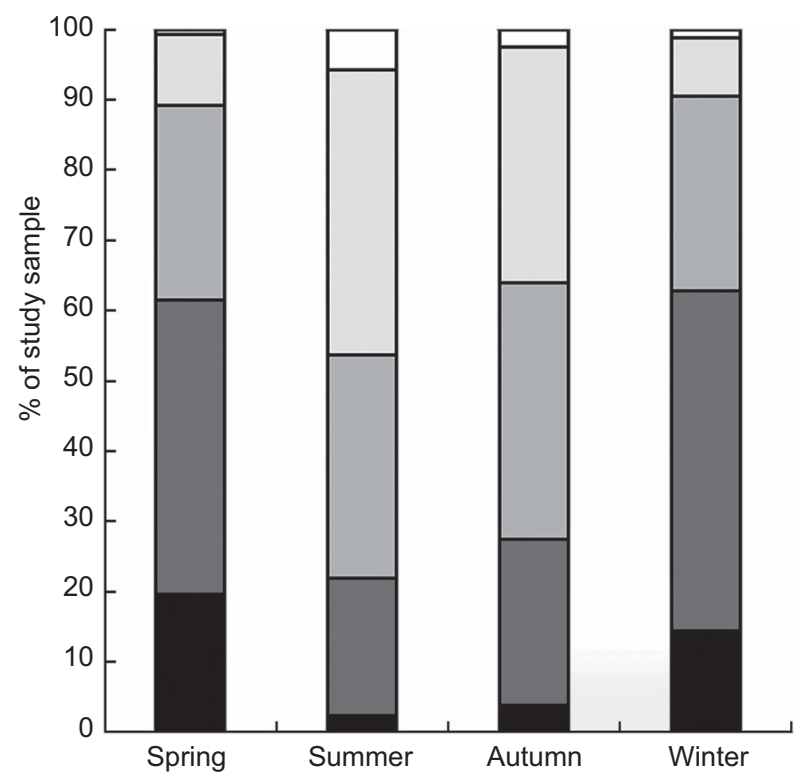

Fig. 1 Percentage of the study sample with serum 25hydroxyvitamin $\mathrm{D}$ concentrations $<10 \mathrm{ng} / \mathrm{ml}(\square), 10-<15 \mathrm{ng} / \mathrm{ml}$ ( $\square$ ), $15-<20 \mathrm{ng} / \mathrm{ml}(\square), 20-<30 \mathrm{ng} / \mathrm{ml}(\square)$ and $\geq 30 \mathrm{ng} / \mathrm{ml}(\square)$ according to season: adolescents aged 12-18 years ( $n$ 1510), Korea National Health and Nutrition Examination Survey (KNHANES) 2008-2009 
Table 1 Characteristics of the study sample according to quartile of serum 25-hydroxyvitamin $D$ concentration: adolescents aged 12-18 years $(n$ 1510), Korea National Health and Nutrition Examination Survey (KNHANES) 2008-2009

\begin{tabular}{|c|c|c|c|c|c|c|c|c|c|c|c|}
\hline & \multirow{2}{*}{\multicolumn{2}{|c|}{ Unweighted }} & \multicolumn{8}{|c|}{ Quartile of 25-hydroxyvitamin D } & \multirow[b]{3}{*}{$P$ value } \\
\hline & & & \multicolumn{2}{|c|}{$<12 \mathrm{ng} / \mathrm{ml}$} & \multicolumn{2}{|c|}{$12-<16 \mathrm{ng} / \mathrm{ml}$} & \multicolumn{2}{|c|}{$16-<20 \mathrm{ng} / \mathrm{ml}$} & \multicolumn{2}{|c|}{$\geq 20 \mathrm{ng} / \mathrm{ml}$} & \\
\hline & $\begin{array}{c}n \text { or } \\
\text { Mean }\end{array}$ & $\begin{array}{c}\% \text { or } \\
\text { SE }\end{array}$ & $\begin{array}{c}n \text { or } \\
\text { Mean }\end{array}$ & $\begin{array}{c}\% \text { or } \\
\text { SE }\end{array}$ & $\begin{array}{c}n \text { or } \\
\text { Mean }\end{array}$ & $\begin{array}{c}\% \text { or } \\
\text { SE }\end{array}$ & $\begin{array}{c}n \text { or } \\
\text { Mean }\end{array}$ & $\begin{array}{l}\% \text { or } \\
\text { SE }\end{array}$ & $\begin{array}{c}n \text { or } \\
\text { Mean }\end{array}$ & $\begin{array}{c}\% \text { or } \\
\text { SE }\end{array}$ & \\
\hline Number & \multicolumn{2}{|c|}{1510} & \multicolumn{2}{|c|}{345} & \multicolumn{2}{|c|}{392} & \multicolumn{2}{|c|}{373} & \multicolumn{2}{|c|}{400} & \\
\hline Age (years), mean (SE) & \multicolumn{2}{|c|}{1510} & $15 \cdot 3$ & $0 \cdot 1$ & $14 \cdot 9$ & $0 \cdot 1$ & $14 \cdot 7$ & $0 \cdot 1$ & $14 \cdot 2$ & $0 \cdot 1$ & $<0.001 \ddagger$ \\
\hline Sex, $n(\%)$ & \multicolumn{2}{|c|}{1510} & & & & & & & & & \\
\hline Males & 806 & $53 \cdot 4$ & 163 & $47 \cdot 2$ & 193 & $49 \cdot 2$ & 216 & $57 \cdot 9$ & 234 & $58 \cdot 5$ & $0 \cdot 197$ \\
\hline Females & 704 & $46 \cdot 6$ & 182 & $52 \cdot 8$ & 199 & $50 \cdot 8$ & 157 & $42 \cdot 1$ & 166 & $41 \cdot 5$ & \\
\hline Season, $n(\%)$ & \multicolumn{2}{|c|}{1510} & & & & & & & & & \\
\hline Winter & 401 & $26 \cdot 6$ & 144 & $41 \cdot 7$ & 133 & 33.9 & 86 & $23 \cdot 1$ & 38 & $9 \cdot 5$ & $<0.001$ \\
\hline Spring & 322 & $21 \cdot 3$ & 128 & $37 \cdot 1$ & 92 & $23 \cdot 5$ & 67 & $18 \cdot 0$ & 35 & $8 \cdot 8$ & \\
\hline Summer & 419 & $27 \cdot 7$ & 38 & $11 \cdot 0$ & 70 & $17 \cdot 9$ & 117 & $31 \cdot 4$ & 194 & $48 \cdot 5$ & \\
\hline Autumn & 368 & $24 \cdot 4$ & 35 & $10 \cdot 1$ & 97 & $27 \cdot 4$ & 103 & $27 \cdot 6$ & 133 & $33 \cdot 3$ & \\
\hline Height Z-score, mean (SE) & \multicolumn{2}{|c|}{1507} & 0.58 & 0.06 & 0.55 & 0.05 & 0.72 & 0.05 & $0 \cdot 60$ & 0.05 & $0.393 \ddagger$ \\
\hline BMI Z-score, mean (SE) & \multicolumn{2}{|c|}{1506} & -0.03 & 0.06 & 0.09 & 0.06 & $0 \cdot 10$ & 0.05 & -0.05 & 0.05 & $0 \cdot 815 \ddagger$ \\
\hline Degree of obesity, $n(\%)$ & \multicolumn{2}{|c|}{1506} & & & & & & & & & \\
\hline Lean & 1234 & $81 \cdot 9$ & 289 & $84 \cdot 3$ & 307 & $78 \cdot 3$ & 300 & $80 \cdot 4$ & 338 & $84 \cdot 9$ & 0.084 \\
\hline Overweight & 184 & $12 \cdot 2$ & 32 & $9 \cdot 3$ & 54 & $13 \cdot 8$ & 47 & $12 \cdot 6$ & 51 & $12 \cdot 8$ & \\
\hline Obese & 88 & $5 \cdot 9$ & 22 & $6 \cdot 4$ & 31 & $7 \cdot 9$ & 26 & $7 \cdot 0$ & 9 & $2 \cdot 3$ & \\
\hline Education level reached, $n(\%)$ & \multicolumn{2}{|c|}{1459} & & & & & & & & & \\
\hline College student & 57 & $3 \cdot 9$ & 15 & $4 \cdot 6$ & 16 & $4 \cdot 3$ & 16 & $4 \cdot 3$ & 10 & $2 \cdot 6$ & $<0.001$ \\
\hline High-school student & 546 & $37 \cdot 4$ & 157 & $48 \cdot 2$ & 153 & $40 \cdot 9$ & 132 & $35 \cdot 8$ & 104 & $26 \cdot 7$ & \\
\hline Middle-school student & 733 & $50 \cdot 2$ & 135 & $41 \cdot 4$ & 186 & $49 \cdot 7$ & 199 & $53 \cdot 9$ & 213 & $54 \cdot 6$ & \\
\hline Elementary-school student & 123 & 8.4 & 19 & $5 \cdot 8$ & 19 & $5 \cdot 1$ & 22 & $6 \cdot 0$ & 63 & $16 \cdot 2$ & \\
\hline Regular physical activity*, $n(\%)$ & \multicolumn{2}{|c|}{1496} & & & & & & & & & \\
\hline Yes & 853 & $57 \cdot 0$ & 158 & $48 \cdot 5$ & 222 & $57 \cdot 1$ & 230 & $62 \cdot 0$ & 243 & $61 \cdot 7$ & 0.031 \\
\hline No & 643 & $43 \cdot 0$ & 184 & $51 \cdot 5$ & 167 & $42 \cdot 9$ & 141 & $38 \cdot 0$ & 151 & $38 \cdot 3$ & \\
\hline Ca intake $(\mathrm{mg} / \mathrm{d})+, n(\%)$ & \multicolumn{2}{|c|}{1269} & & & & & & & & & \\
\hline$\geq \mathrm{DRI}$ & 123 & $9 \cdot 7$ & 25 & $8 \cdot 7$ & 28 & $9 \cdot 3$ & 34 & $10 \cdot 9$ & 36 & $10 \cdot 6$ & 0.681 \\
\hline$<\mathrm{DRI}$ & 1146 & $90 \cdot 3$ & 264 & $91 \cdot 3$ & 300 & $90 \cdot 7$ & 278 & $99 \cdot 1$ & 304 & $99 \cdot 4$ & \\
\hline Vitamin D supplement use, $n(\%)$ & \multicolumn{2}{|c|}{1147} & & & & & & & & & \\
\hline Yes & 95 & $8 \cdot 3$ & 14 & $4 \cdot 6$ & 26 & $7 \cdot 4$ & 28 & $8 \cdot 3$ & 27 & $10 \cdot 8$ & 0.028 \\
\hline$\geq 5 \mu \mathrm{g} / \mathrm{d}$ & 35 & $3 \cdot 1$ & 3 & $1 \cdot 0$ & 12 & $3 \cdot 4$ & 12 & $3 \cdot 6$ & 8 & $3 \cdot 2$ & \\
\hline $0-<5 \mu \mathrm{g} / \mathrm{d}$ & 60 & $5 \cdot 2$ & 11 & $3 \cdot 6$ & 14 & $4 \cdot 0$ & 16 & $4 \cdot 7$ & 19 & $7 \cdot 6$ & \\
\hline No & 1052 & $91 \cdot 7$ & 275 & $90 \cdot 8$ & 301 & $85 \cdot 3$ & 281 & $83 \cdot 4$ & 195 & $78 \cdot 3$ & \\
\hline Milk intake (ml/d), $n(\%)$ & \multicolumn{2}{|c|}{1219} & & & & & & & & & \\
\hline$\geq 400 \mathrm{ml} / \mathrm{d}$ & 92 & $7 \cdot 5$ & 19 & $6 \cdot 9$ & 12 & $3 \cdot 8$ & 29 & $9 \cdot 6$ & 32 & $9 \cdot 8$ & 0.002 \\
\hline $200-<400 \mathrm{ml} / \mathrm{d}$ & 364 & $29 \cdot 9$ & 75 & $27 \cdot 2$ & 78 & $24 \cdot 7$ & 102 & $33 \cdot 8$ & 109 & $33 \cdot 5$ & \\
\hline $0-<200 \mathrm{ml} / \mathrm{d}$ & 763 & $62 \cdot 6$ & 182 & $65 \cdot \overline{9}$ & 226 & $71 \cdot 5$ & 171 & $55 \cdot 6$ & 184 & $56 \cdot 6$ & \\
\hline Alkaline phosphatase (IU/I), mean (SE) & & & 585 & $24 \cdot 9$ & 512 & $20 \cdot 5$ & 503 & $20 \cdot 4$ & 473 & $22 \cdot 7$ & $<0.001 \ddagger$ \\
\hline
\end{tabular}

DRI, Dietary Recommended Intake.

Data are presented as numbers and percentages or means with their standard errors, as indicated.

The Rao-Scott $\chi^{2}$ test was used to analyse associations between quartiles of serum 25-hydroxyvitamin D and categorical variables.

${ }^{*}$ Regular physical activity: participants who performed moderate or vigorous physical activity for at least $60 \mathrm{~min} / \mathrm{d}$ on $3 \mathrm{~d} / \mathrm{week}$, and/or walking activity for at least 60 mind on $5 \mathrm{~d} /$ week, and/or muscular strength activity on more than $3 \mathrm{~d} /$ week were assigned to the 'regular physical activity' group. Those participants who performed moderate or vigorous physical activity for at least $180 \mathrm{~min} / \mathrm{d}$ on $3 \mathrm{~d} /$ week were also assigned to the 'regular physical activity' group.

tThe recommended DRI is $1000 \mathrm{mg} / \mathrm{d}$ for boys aged $12-14$ years, $900 \mathrm{mg} / \mathrm{d}$ for girls aged $12-14$ years, $900 \mathrm{mg} / \mathrm{d}$ for boys aged $15-18$ years and $800 \mathrm{mg} / \mathrm{d}$ for girls aged $15-18$ years.

$\ddagger W e$ estimated means with their standard errors for age, height, BMI Z-score and serum alkaline phosphatase levels according to the quartiles and examined these associations with a test for linear trend.

the dependent variable. Older age group $(P<0 \cdot 001)$, female gender $(P=0 \cdot 006)$ and a lack of physical activity $(P=0 \cdot 002)$ were significantly associated with low vitamin D status. Obese participants had a lower vitamin D status than did those who were lean $(P=0 \cdot 048)$ or overweight $(P=0 \cdot 021)$. Participants who were not taking supplemental vitamin $\mathrm{D}$ had a lower vitamin $\mathrm{D}$ status than those who were taking $0-<5 \mu \mathrm{g} / \mathrm{d}(P=0 \cdot 043)$ and those who were taking $\geq 5 \mu \mathrm{g} / \mathrm{d}(P=0 \cdot 003)$. Participants who consumed $\geq 400 \mathrm{ml} \mathrm{milk/d}$ had a higher vitamin $D$ status than those who consumed $0-<200 \mathrm{ml} / \mathrm{d} \quad(P=0 \cdot 029)$. The interaction of season with the effect of vitamin $D$ supplement use on vitamin D status was statistically significant $(P=0 \cdot 040)$.

Multiple linear regression analysis showed that season $(P<0 \cdot 001)$, education level $(P<0 \cdot 001)$ and vitamin $\mathrm{D}$ supplement use $(P=0 \cdot 012)$ were independent predictors for vitamin D status (Table 2). In addition, the modifying effect of season on the association between vitamin D supplement use and vitamin D status was significant $(P<0 \cdot 001 ;$ Table 2, Fig. 2). Finally, multiple linear regression analyses were performed separately for each season (Table 3$)$. During the winter, gender $(P=0 \cdot 005)$, education level $(P=0 \cdot 011)$, regular physical activity 
Table 2 Results of unadjusted and multivariate-adjusted linear regression analyses with log-transformed 25-hydroxyvitamin D concentrations as the dependent variable $\left(R^{2}=0.274\right)$ : adolescents aged 12-18 years, Korea National Health and Nutrition Examination Survey (KNHANES) 2008-2009

\begin{tabular}{|c|c|c|c|c|c|c|c|c|}
\hline & \multicolumn{4}{|c|}{ Unadjusted ( $n$ 1510) } & \multicolumn{4}{|c|}{ Multivariate-adjusted ( $n$ 1056) } \\
\hline & $\beta$ & SE & $95 \% \mathrm{Cl}$ & $P$ value & $\beta$ & SE & $95 \% \mathrm{Cl}$ & $P$ value \\
\hline Constant & - & - & - & - & $2 \cdot 656$ & 0.057 & $2 \cdot 543,2 \cdot 769$ & $<0.001$ \\
\hline Age $^{*}$ & -0.041 & 0.005 & $-0.052,-0.030$ & $<0.001$ & - & - & - & - \\
\hline \multicolumn{9}{|c|}{ - } \\
\hline Males ( $v$. females) & 0.058 & 0.021 & $0.017,0.098$ & 0.006 & 0.035 & 0.020 & $-0.004,0.075$ & 0.080 \\
\hline Season ( $v$. winter) & & & & $<0.001$ & & & & $<0.001$ \\
\hline Spring & -0.009 & 0.033 & $-0.074,0.055$ & $0 \cdot 774$ & $0 \cdot 010$ & 0.034 & $-0.056,0.076$ & $0 \cdot 757$ \\
\hline Summer & 0.335 & 0.031 & $0 \cdot 274,0.397$ & $<0.001$ & $0 \cdot 314$ & 0.036 & $0.243,0.384$ & $<0.001$ \\
\hline Autumn & $0 \cdot 277$ & 0.030 & $0.218,0.336$ & $<0.001$ & $0 \cdot 257$ & 0.032 & $0 \cdot 194,0 \cdot 320$ & $<0.001$ \\
\hline Degree of obesity ( $v$. obese) & & & & 0.043 & & & & $0 \cdot 100$ \\
\hline Lean & 0.072 & 0.036 & $0,0.143$ & 0.048 & 0.063 & 0.033 & $-0.003,0.128$ & 0.060 \\
\hline Overweight & 0.098 & 0.042 & $0 \cdot 015,0 \cdot 182$ & 0.021 & 0.075 & 0.038 & $-0.002,0.151$ & 0.050 \\
\hline $\begin{array}{l}\text { Education level reached } \\
\text { ( } v . \text { elementary-school student) }\end{array}$ & & & & $<0.001$ & & & & $<0.001$ \\
\hline College student & -0.228 & 0.060 & $-0.346,-0.111$ & $<0.001$ & -0.256 & 0.065 & $-0.384,-0.127$ & $<0.001$ \\
\hline High-school student & -0.228 & 0.038 & $-0.302,-0.153$ & $<0.001$ & -0.202 & 0.040 & $-0.280,-0.124$ & $<0.001$ \\
\hline Middle-school student & $-0 \cdot 111$ & 0.038 & $-0.185,-0.037$ & 0.003 & -0.098 & 0.039 & $-0.175,-0.020$ & 0.014 \\
\hline \multicolumn{9}{|l|}{ Regular physical activity† ( $v$. yes) } \\
\hline No & -0.067 & 0.021 & $-0.109,-0.025$ & 0.002 & -0.037 & 0.020 & $-0.076,0.002$ & 0.060 \\
\hline Vitamin D supplement use ( $v$. no) & & & & 0.006 & & & & 0.012 \\
\hline$\geq 5 \mu \mathrm{g} / \mathrm{d}$ & $0 \cdot 146$ & 0.049 & $0 \cdot 049,0 \cdot 243$ & 0.003 & 0.259 & $0 \cdot 047$ & $0 \cdot 166,0 \cdot 352$ & $<0.001$ \\
\hline $0-<5 \mu \mathrm{g} / \mathrm{d}$ & $0 \cdot 118$ & 0.058 & $0.004,0.233$ & 0.043 & 0.249 & 0.087 & $0.077,0.421$ & 0.005 \\
\hline Milk intake $(v . \geq 400 \mathrm{ml} / \mathrm{d})$ & & & & 0.058 & & & & 0.591 \\
\hline $0-<200 \mathrm{ml} / \mathrm{d}$ & $-0 \cdot 105$ & 0.048 & $-0.200,-0.011$ & 0.029 & -0.035 & 0.034 & $-0.101,0.031$ & 0.295 \\
\hline $200-<400 \mathrm{ml} / \mathrm{d}$ & -0.309 & 0.051 & $-0 \cdot 138,0.060$ & $0 \cdot 441$ & -0.001 & 0.036 & $-0.070,0.071$ & 0.978 \\
\hline $\begin{array}{l}\text { Interaction between season and vitamin D } \\
\text { supplements (season } \times \text { supplements) } \ddagger\end{array}$ & - & - & - & - & - & - & - & $<0.001$ \\
\hline \multicolumn{9}{|l|}{$\mathrm{Ca}$ intake $(\mathrm{mg} / \mathrm{d}) \S(v .<\mathrm{DRI})$} \\
\hline$\geq \mathrm{DRI}$ & 0.006 & 0.031 & $-0.066,0.055$ & $0 \cdot 856$ & - & - & - & - \\
\hline
\end{tabular}

DRI, Dietary Recommended Intake.

${ }^{*}$ Age was excluded in multivariate-adjusted linear regression analysis because of collinearity with the education level reached.

tRegular physical activity: participants who performed moderate or vigorous physical activity for at least $60 \mathrm{~min} / \mathrm{d}$ on $3 \mathrm{~d} / \mathrm{week}$, and/or walking activity for at least $60 \mathrm{~min} / \mathrm{d}$ on $5 \mathrm{~d} /$ week, and/or muscular strength activity on more than $3 \mathrm{~d} /$ week were assigned to the 'regular physical activity' group. Those participants who performed moderate or vigorous physical activity for at least $180 \mathrm{~min} / \mathrm{d}$ on $3 \mathrm{~d} /$ week were also assigned to the 'regular physical activity' group.

$\ddagger$ The modifying effect of season on the association between vitamin $D$ supplement use and vitamin $D$ status was significant in unadjusted and multivariateadjusted linear regression analyses.

$\S$ The recommended DRI is $1000 \mathrm{mg} / \mathrm{d}$ for boys aged $12-14$ years, $900 \mathrm{mg} / \mathrm{d}$ for girls aged $12-14$ years, $900 \mathrm{mg} / \mathrm{d}$ for boys aged $15-18$ years and $800 \mathrm{mg} / \mathrm{d}$ for girls aged $15-18$ years.

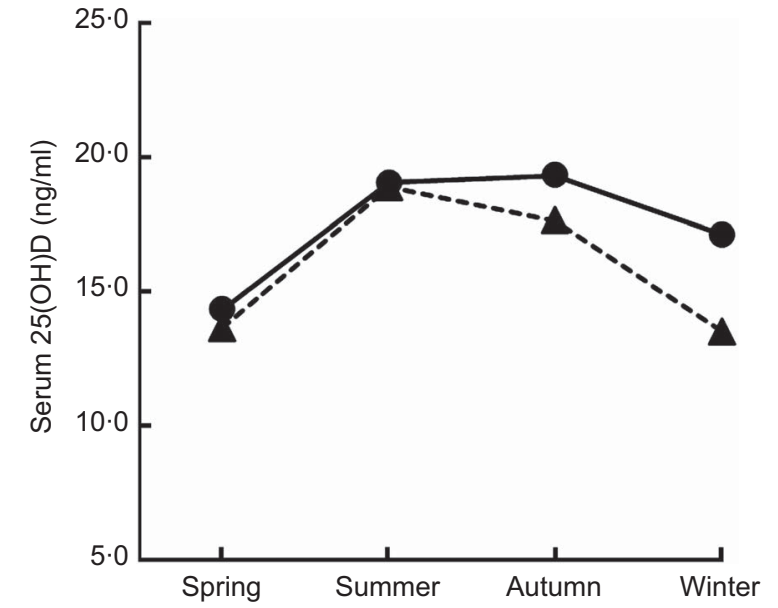

Fig. 2 The modifying effect of season on the association between vitamin D supplement use $(--\boldsymbol{\Delta}--$, supplement use $=$ no; - - , supplement use $=$ yes) and vitamin D status (measured as serum 25 -hydroxyvitamin $\mathrm{D}(25(\mathrm{OH}) \mathrm{D})$ concentration $(\mathrm{ng} / \mathrm{ml})$ ) was significant $(P<0.001)$, after adjustments were made for other variables: adolescents aged $12-18$ years ( $n$ 1510), Korea National Health and Nutrition Examination Survey (KNHANES) 2008-2009
$(P=0 \cdot 011)$ and vitamin D supplement use $(P<0 \cdot 001)$ were significantly associated with vitamin $\mathrm{D}$ status.

\section{Discussion}

More than half of these Korean adolescents showed a serum 25(OH)D level of $<20 \mathrm{ng} / \mathrm{ml}$, even in summer and autumn. The prevalence of vitamin D deficiency reached about $90 \%$ during winter and spring. There was a lower seasonal variation in serum $25(\mathrm{OH}) \mathrm{D}$ concentrations in participants who were taking vitamin $\mathrm{D}$ supplements than in those who were not. Winter season, higher education level and a lack of vitamin D supplementation were independent predictors for low vitamin D status in these Korean adolescents.

The concentrations of serum $25(\mathrm{OH}) \mathrm{D}$ were highest in summer and lowest in winter and spring. According to 2008-2009 data of the Korean Meteorological Administration, the shortest duration of sunshine is observed between November and March and between late June 
Table 3 Results of multivariate-adjusted linear regression analyses with log-transformed 25-hydroxyvitamin D concentrations as the dependent variable separately for each season: adolescents aged 12-18 years ( $n$ 1056), Korea National Health and Nutrition Examination Survey (KNHANES) 2008-2009

\begin{tabular}{|c|c|c|c|c|c|c|c|c|c|c|c|c|}
\hline & \multicolumn{3}{|c|}{ Spring (n 223) } & \multicolumn{3}{|c|}{ Summer (n 256) } & \multicolumn{3}{|c|}{ Autumn ( $n$ 264) } & \multicolumn{3}{|c|}{ Winter ( $n$ 313) } \\
\hline & $\beta$ & SE & $P$ value & $\beta$ & SE & $P$ value & $\beta$ & SE & $P$ value & $\beta$ & SE & $P$ value \\
\hline $\begin{array}{l}\text { Constant } \\
\text { Sex ( } v \text {. females) }\end{array}$ & $2 \cdot 734$ & $0 \cdot 160$ & $<0.001$ & $3 \cdot 081$ & $0 \cdot 110$ & $<0.001$ & $2 \cdot 823$ & 0.093 & $<0.001$ & $2 \cdot 699$ & 0.013 & $<0.001$ \\
\hline Males & 0.017 & 0.047 & 0.723 & -0.006 & 0.043 & 0.890 & 0.060 & 0.039 & 0.125 & 0.071 & 0.024 & 0.005 \\
\hline Degree of obesity ( $v$. obese) & & & 0.554 & & & $0 \cdot 144$ & & & $0 \cdot 315$ & & & $0 \cdot 882$ \\
\hline Lean & 0.065 & 0.059 & $0 \cdot 277$ & $0 \cdot 127$ & 0.069 & 0.072 & 0.054 & 0.048 & $0 \cdot 270$ & 0.003 & 0.058 & 0.963 \\
\hline Overweight & 0.074 & 0.088 & 0.406 & $0 \cdot 122$ & 0.077 & $0 \cdot 119$ & 0.072 & 0.050 & $0 \cdot 157$ & 0.019 & 0.064 & 0.765 \\
\hline $\begin{array}{l}\text { Education reached } \\
(v . \text { elementary-school student) }\end{array}$ & & & $0 \cdot 214$ & & & $<0.001$ & & & 0.005 & & & 0.011 \\
\hline College student & -0.275 & 0.149 & 0.071 & -0.452 & 0.094 & $<0.001$ & -0.074 & $0 \cdot 166$ & 0.658 & $-0 \cdot 122$ & $0 \cdot 138$ & 0.383 \\
\hline High & $-0 \cdot 172$ & $0 \cdot 124$ & $0 \cdot 114$ & $-0 \cdot 300$ & 0.093 & 02 & $-0 \cdot 214$ & 0.0 & 20 & $-0 \cdot 184$ & 0 . & 04 \\
\hline Middle-school student & -0.064 & $0 \cdot 125$ & 0.611 & -0.288 & 0.084 & 0.001 & -0.052 & 0.065 & $0 \cdot 430$ & -0.070 & 0.058 & 0.235 \\
\hline Regular physical activity* & & & & & & & & & & & & \\
\hline No & -0.041 & 0.045 & 0.375 & -0.051 & 0.041 & $0 \cdot 217$ & -0.009 & 0.036 & $0 \cdot 796$ & -0.089 & 0.034 & 0.011 \\
\hline Vitamin D supplement use ( $v$. no) & & & 0.929 & & & 0.067 & & & 0.039 & & & $<0.001$ \\
\hline $0-<5 \mu \mathrm{g} / \mathrm{d}$ & 0.028 & 0.013 & $0 \cdot 807$ & $0 \cdot 182$ & 0.084 & 0.034 & 0.030 & 0.0 & $0 \cdot 734$ & $0 \cdot 276$ & 0.047 & $<0.001$ \\
\hline$\geq 5$ & -0.025 & 0.081 & 0.760 & 0.095 & 0.049 & 0.059 & 0.207 & 0.086 & 0.020 & 0.250 & 0.085 & 0.005 \\
\hline Milk in & & & 0.335 & & & 0.726 & & & 0.924 & & & 0.836 \\
\hline $0-<200 \mathrm{ml} / \mathrm{d}$ & $-0 \cdot 106$ & 0.076 & $0 \cdot 168$ & -0.035 & 0.038 & 0.363 & 0.014 & 0.055 & $0 \cdot 690$ & -0.060 & 0.071 & 0.399 \\
\hline $200-<400 \mathrm{ml} / \mathrm{d}$ & -0.079 & 0.072 & $0 \cdot 280$ & -0.001 & 0.052 & 0.980 & 0.013 & 0.061 & 0.756 & 0.040 & 0.076 & 0.603 \\
\hline
\end{tabular}

${ }^{*}$ Regular physical activity: participants who performed moderate or vigorous physical activity for at least $60 \mathrm{~min} / \mathrm{d}$ on $3 \mathrm{~d} / \mathrm{week}$, and/or walking activity for at least $60 \mathrm{~min} / \mathrm{d}$ on $5 \mathrm{~d} /$ week, and/or muscular strength activity on more than $3 \mathrm{~d} /$ week were assigned to the 'regular physical activity' group. Those participants who performed moderate or vigorous physical activity for at least $180 \mathrm{~min} / \mathrm{d}$ on $3 \mathrm{~d} /$ week were also assigned to the 'regular physical activity' group.

and July ${ }^{(19)}$. Despite the longest duration of sunshine being observed between April and May, the lowest level of serum $25(\mathrm{OH}) \mathrm{D}$ seen during winter had not yet recovered by spring. Additionally, the unexpectedly high prevalence of vitamin D deficiency in summer might be attributed to the short duration of sunshine between late June and July, because of the rainy season and heavy cloud cover at that time of year ${ }^{(19)}$. This high prevalence of vitamin D deficiency in Korean adolescents is consistent with previous observations including subjects of similar age groups living at latitudes around $37^{\circ} \mathrm{N}$. For example, there was a $45 \%$ prevalence in young women aged $16-22$ years residing in California $\left(38^{\circ} \mathrm{N}\right)$ between May and October ${ }^{(20)}$ and an $89 \cdot 2 \%$ prevalence among girls aged $15(\mathrm{SD} 0 \cdot 4)$ years in Beijing $\left(40^{\circ} \mathrm{N}\right)$ in winter ${ }^{(12)}$.

The age-related decline in the serum 25(OH)D level might be explained by unmeasured factors such as sunlight exposure, the change in fat mass and increased nutrient needs during growth ${ }^{(21)}$. Older age and higher education level were both associated with low vitamin D status. The level of schooling could affect vitamin D status through differences in sunlight exposure with differing amounts of outdoor activity. With increasing school grade, the total amount of time spent indoors in a classroom increases and participation in after-school outdoor activities decreases ${ }^{(22)}$. The prevalence of vitamin D deficiency throughout the year reached approximately $70 \%, 80 \%$ and $83 \%$ in middle school, high school and college students, respectively. According to a recent report ${ }^{(23)}$, most American children in both southern $\left(35^{\circ} \mathrm{N}\right)$ and northern $\left(45^{\circ} \mathrm{N}\right)$ regions may not be getting adequate outdoor UVB exposures to satisfy their vitamin D needs all year. Although our study was limited because UVB exposure was not recorded in the KNHANES data, we assumed that decreased after-school daylight hours for participants enrolled in higher school grades might contribute to insufficient UVB exposure and low vitamin D status.

We hypothesized that vitamin D intake is an important predictor of low vitamin D status. Unfortunately, the KNHANES database does not detail total daily vitamin D intake in foods and supplements. Thus, we included two dietary factors in our analysis: vitamin D supplementation and milk consumption, although this is not enough to assess total daily vitamin D intake. Milk consumption was an unadjusted risk factor for low vitamin D status, but was not a multivariate-adjusted risk factor for low vitamin D status. This may be attributable to the fact that most Korean adolescents consume non-fortified milk, rather than fortified milk ${ }^{(24)}$. Milk intake in the average Korean household is very low and most Korean adolescents drink non-fortified milk that is provided in their school lunches ${ }^{(24)}$, which contains $0 \cdot 25 \mu \mathrm{g}$ of cholecalcidiol/ $100 \mathrm{ml}^{(25)}$. However, vitamin D supplementation was an independent predictor for a better vitamin $\mathrm{D}$ status. The seasonal variation in serum $25(\mathrm{OH}) \mathrm{D}$ concentration was smaller in participants who were taking vitamin D supplements than in those who were not. Whiting et al. ${ }^{(26)}$ recently determined the prevalence of meeting the DRI and the role of vitamin D supplement use among Canadians aged 6-79 years. Vitamin D supplement users had significantly higher $25(\mathrm{OH}) \mathrm{D}$ concentrations than did non-users, and no seasonal differences were found ${ }^{(26)}$. In Korean adolescents living between latitudes $33^{\circ}$ and $39^{\circ} \mathrm{N}$, the use of vitamin D supplements may contribute to 
maintain a better $25(\mathrm{OH}) \mathrm{D}$ status with lower seasonal variation.

In our study, $90 \%$ of obese adolescents were vitamin D deficient, which is consistent with recent observations reporting high rates of vitamin $\mathrm{D}$ deficiency in obese individuals $^{(22,27)}$. Despite a marginal statistical significance, obese adolescents independently had a lower vitamin D status than lean or overweight adolescents in our analysis. Recent studies reported an independent inverse relationship between serum 25(OH)D concentrations and adiposity measurements, including BMI percentiles, total fat mass and visceral adipose tissue, after adjustment for covariates ${ }^{(9,21)}$. Adipose tissue can sequester lipid-soluble vitamin D, leading to reduced bioavailability. However, whether adiposity per se lowers vitamin D levels is still controversial.

Whereas the estimated Adequate Intake for vitamin D was $5 \mu \mathrm{g} / \mathrm{d}$ in 1997, the vitamin D Estimated Average Requirement and RDA published by the US Institute of Medicine in 2010 were increased to $10 \mu \mathrm{g} / \mathrm{d}$ and $15 \mu \mathrm{g} / \mathrm{d}$ to give $25(\mathrm{OH}) \mathrm{D}$ concentrations of $16 \mathrm{ng} / \mathrm{ml}$ and $20 \mathrm{ng} / \mathrm{ml}$, respectively $^{(3)}$. In strong contrast to these recommendations, the Korean Nutrition Society lowered the DRI to $5 \mu \mathrm{g} / \mathrm{d}$ in $2010^{(14)}$, based on a small amount of evidence about the effect of vitamin D on health outcomes in Korean adolescents and the assumption that exposure to sunlight guarantees sufficient vitamin D status. However, the high prevalence of vitamin $\mathrm{D}$ deficiency raises the question of whether daylight outdoor hours and/or the current 2010 DRI of vitamin D are enough to maintain a sufficient vitamin D status in Korean adolescents.

The present study was limited by a lack of information about daylight hours, use of sunscreen in the summer season, total vitamin $\mathrm{D}$ intake and total body and/or visceral adiposity. Moreover, supporting variables related to outcomes of vitamin D deficiency such as serum intact parathyroid hormone levels or bone parameters were not measured. Although the Rural Development Administration food composition tables provide the vitamin $\mathrm{D}$ content of foods ${ }^{(24)}$, there is no comprehensive nutrient database or validated FFQ in Korea. Although our study cannot make any recommendation regarding current DRI for daily vitamin D intake in Korean adolescents, these limitations need to be addressed in further studies to investigate accurate assessment of total daily vitamin D intake and its relationship with 25(OH)D and parathyroid hormone concentrations and health outcomes.

\section{Conclusion}

Vitamin D deficiency was highly prevalent in Korean adolescents, especially those in higher school grades. More than half of them were vitamin D deficient, even in summer. Winter season, higher education level and a lack of vitamin D supplementation were independent risk factors for low vitamin D status. Vitamin D supplementation may contribute to maintain a better vitamin D status with lower seasonal variation. Further studies are required to determine optimal vitamin $\mathrm{D}$ intakes to maintain sufficient vitamin D status for Korean adolescents.

\section{Acknowledgements}

Sources of funding: This research received no specific grant from any funding agency in the public, commercial or not-for-profit sectors. Conflicts of interest: All authors have no conflicts of interest. Authors' contributions: Y.A.L., H.Y.K., S.W.Y. and C.H.S. were responsible for the conception and design of the study. Y.A.L., H.H., J.Y.K. and H.J.K. contributed to prepare the data set. Y.A.L. and H.H. performed the statistical analysis. Y.A.L. and C.H.S. drafted the manuscript. All authors played a role in interpretation of the results and approved the final manuscript.

\section{References}

1. Adams JS \& Hewison M (2010) Update in vitamin D. J Clin Endocrinol Metab 95, 471-478.

2. Holick MF \& Chen TC (2008) Vitamin D deficiency: a worldwide problem with health consequences. Am J Clin Nutr 87, issue 4, 1080S-1086S.

3. Ross AC, Manson JE, Abrams SA et al. (2011) The 2011 report on dietary reference intakes for calcium and vitamin $\mathrm{D}$ from the Institute of Medicine: what clinicians need to know. J Clin Endocrinol Metab 96, 53-58.

4. Rosen CJ (2011) Clinical practice. Vitamin D insufficiency. $N$ Engl J Med 364, 248-254.

5. Holick MF, Binkley NC, Bischoff-Ferrari HA et al. (2011) Evaluation, treatment, and prevention of vitamin D deficiency: an Endocrine Society clinical practice guideline. J Clin Endocrinol Metab 96, 1911-1930.

6. Kumar J, Muntner P, Kaskel FJ et al. (2009) Prevalence and associations of 25-hydroxyvitamin D deficiency in US children: NHANES 2001-2004. Pediatrics 124, e362-e370.

7. Gordon CM, DePeter KC, Feldman HA et al. (2004) Prevalence of vitamin D deficiency among healthy adolescents. Arch Pediatr Adolesc Med 158, 531-537.

8. Weng FL, Shults J, Leonard MB et al. (2007) Risk factors for low serum 25-hydroxyvitamin D concentrations in otherwise healthy children and adolescents. Am J Clin Nutr 86, 150-158.

9. Dong Y, Pollock N, Stallmann-Jorgensen IS et al. (2010) Low 25-hydroxyvitamin $\mathrm{D}$ levels in adolescents: race, season, adiposity, physical activity, and fitness. Pediatrics 125, 1104-1111.

10. Rockell JE, Green TJ, Skeaff CM et al. (2005) Season and ethnicity are determinants of serum 25-hydroxyvitamin D concentrations in New Zealand children aged 5-14 years. J Nutr 135, 2602-2608.

11. Absoud M, Cummins C, Lim MJ et al. (2011) Prevalence and predictors of vitamin D insufficiency in children: a Great Britain population based study. PLoS One 6, e22179.

12. Foo LH, Zhang Q, Zhu K et al. (2009) Relationship between vitamin $\mathrm{D}$ status, body composition and physical exercise in adolescent girls in Beijing. Osteoporos Int 20, 417-425.

13. Choi HS, Oh HJ, Choi H et al. (2011) Vitamin D insufficiency in Korea - a greater threat to younger generation: the Korea National Health and Nutrition Examination Survey (KNHANES) 2008. J Clin Endocrinol Metab 96, 643-651.

14. Ryoo E (2011) Adolescent nutrition: what do pediatricians do? Korean J Pediatr 54, 287-291. 
15. Moon JS, Lee SY, Nam CM et al. (2008) 2007 Korean National Growth Charts: review of developmental process and an outlook. Korean J Pediatr 51, 1-25.

16. US Department of Health and Human Services (2008) 2008 Physical Activity Guidelines for Americans: Be Active, Healthy, and Happy! http://www.health.gov/paguidelines (accessed February 2013).

17. Korea Centers for Disease Control and Prevention (2009) The Statistics of 5 th Korea Youth Risk Behavior Web-based Survey (KYRBWS) in 2009. http://yhs.cdc.go.kr/ (accessed March 2011).

18. Sempos CT, Vesper HW, Phinney KW et al. (2012) Vitamin $\mathrm{D}$ status as an international issue: national surveys and the problem of standardization. Scand J Clin Lab Invest Suppl 243, 32-40.

19. Korea Meteorological Administration (2012) 2008-2009 Climatic data. http://www.kma.go.kr/weather/climate/ average_historic.jsp (accessed February 2013).

20. Kremer R, Campbell PP, Reinhardt T et al. (2009) Vitamin D status and its relationship to body fat, final height, and peak bone mass in young women. J Clin Endocrinol Metab 94, 67-73.
21. Olson ML, Maalouf NM, Oden JD et al. (2012) Vitamin D deficiency in obese children and its relationship to glucose homeostasis. J Clin Endocrinol Metab 97, 279-285.

22. Park KW (2006) A study on adolescents' daily leisure activities and use of cultural facilities. Culture Tourism Res 8, 7-24.

23. Godar DE, Pope SJ, Grant WB et al. (2012) Solar UV doses of young Americans and vitamin $\mathrm{D}_{3}$ production. Environ Health Perspect 120, 139-143.

24. National Rural Resources Development Institute (2006) Food composition table, 7th revision. http://lib.rda.go.kr/ newlib/index.asp (accessed June 2012).

25. Kim JH (2007) Analysis of the awareness of the value and the consumption pattern on milk of elementary middle and high school students. J Korean Acad Fam Med 45, 23-33.

26. Whiting SJ, Langlois KA, Vatanparast $\mathrm{H}$ et al. (2011) The vitamin D status of Canadians relative to the 2011 Dietary Reference Intakes: an examination in children and adults with and without supplement use. Am J Clin Nutr 94, 128-135.

27. Alemzadeh R, Kichler J, Babar G et al. (2008) Hypovitaminosis D in obese children and adolescents: relationship with adiposity, insulin sensitivity, ethnicity, and season. Metabolism 57, 183-191. 